\title{
Ultrasonographic changes in the liver tumors as indicators of adequate tumor coverage with electric field for effective electrochemotherapy
}

\author{
Nina Boc ${ }^{1}$, Ibrahim Edhemovic ${ }^{1}$, Bor Kos², Maja M. Music ${ }^{1}$, Erik Brecelj¹, Blaz Trotovsek², \\ Masa Bosnjak ${ }^{1}$, Mihajlo Djokic ${ }^{3}$, Damijan Miklavcic ${ }^{2}$, Maja Cemazar ${ }^{1,4}$, Gregor Sersa ${ }^{1,5}$ \\ ${ }^{1}$ Institute of Oncology Ljubljana, Ljubljana, Slovenia \\ ${ }^{2}$ Faculty of Electrical Engineering, University of Ljubljana, Ljubljana, Slovenia \\ ${ }^{3}$ University Medical Center, Ljubljana, Ljubljana, Slovenia \\ ${ }^{4}$ Faculty of Health Sciences, University of Primorska, Izola, Slovenia \\ ${ }^{5}$ Faculty of Health Sciences, University of Ljubljana, Ljubljana, Slovenia
}

Radiol Oncol 2018; 52(4): 383-391.

Received 3 September 2018

Accepted 4 October 2018

Correspondence to: Prof. Gregor Serša, Ph.D., Institute of Oncology Ljubljana, Zaloska 2, SI-1000 Ljubljana, Slovenia. E-mail: gsersa@onko-i.si

Disclosure: Damijan Miklavčič holds patents on electrochemotherapy that have been licensed to IGEA S.p.a (Carpi, Italy) and is also a consultant to various companies with an interest in electroporation based technologies and treatments. The other authors have no competing interests.

Background. The aim of the study was to characterize ultrasonographic (US) findings during and after electrochemotherapy of liver tumors to determine the actual ablation zone and to verify the coverage of the treated tumor with a sufficiently strong electric field for effective electrochemotherapy.

Patients and methods. US findings from two representative patients that describe immediate and delayed tumor changes after electrochemotherapy of colorectal liver metastases are presented.

Results. The US findings were interrelated with magnetic resonance imaging (MRI). Electrochemotherapy-treated tumors were exposed to electric pulses based on computational treatment planning. The US findings indicate immediate appearance of hyperechogenic microbubbles along the electrode tracks. Within minutes, the tumors became evenly hyperechogenic, and simultaneously, an oedematous rim was formed presenting as a hypoechogenic formation which persisted for several hours after treatment. The US findings overlapped with computed electric field distribution in the treated tissue, indicating adequate coverage of tumors with sufficiently strong electric field, which may predict an effective treatment outcome.

Conclusions. US provides a tool for assessment of appropriate electrode insertion for intraoperative electrochemotherapy of liver tumors and assessment of the appropriate coverage of a tumor with a sufficiently strong electric field and can serve as predictor of the response of tumors.

Key words: electrochemotherapy; ultrasound; treatment plan; colorectal liver metastases

\section{Introduction}

Ablative techniques provide an effective tool for treatment of liver tumors. Radiofrequency ablation is the most frequently used, whereas electropora- tion-based treatments, i.e., electrochemotherapy (ECT) and irreversible electroporation (IRE), are being explored as possible alternatives. ${ }^{1-6}$ The major advantage of electroporation-based treatments over other ablation techniques is their non-thermal 
mechanism of action; therefore, they have the advantage of being effective near major blood vessels, since their effectiveness is not reduced by the heat sink effect. ${ }^{1}$

ECT combines chemotherapeutic drugs, bleomycin or cisplatin, to provide therapeutic effectiveness with electroporation of tumors as a drug delivery system, whereas in IRE, cell death is induced by electroporation only., ${ }^{3,7-9}$ ECT has recently demonstrated its effectiveness in a first clinical study on liver tumors, i.e., colorectal liver metastases during open surgery. ${ }^{2,10,11}$ Effectiveness was demonstrated to be good: $80 \%$ of the treated tumors responded completely during the 4-months observation period. The safety of its use was also demonstrated in tumors located near the major liver blood vessels, which were not resectable and not amenable to radiofrequency ablation. The phase I study is now continuing with a phase II study in which ECT was also demonstrated to be effective in tumors that are larger than $3 \mathrm{~cm}$ in diameter. ${ }^{11}$ Recently similar results were obtained in phase I studi after ECT of hepatocellular carcinoma tumors. ${ }^{12}$

A wide spectrum of imaging techniques is available for the evaluation of the outcome of IRE of liver tumors. ${ }^{13}$ Among these techniques are US, computed tomography (CT), magnetic resonance imaging (MRI) and positron emission tomography (PET). All of these techniques contribute to the planning, treatment implementation, visualization of the target volume, and guidance for the electrode placement. MRI has already been utilized for the immediate observation of ablated tissue zones and monitoring of the IRE ablation procedure. ${ }^{14-16}$

ECT is currently performed intraoperatively, and the US is used for tumor identification and electrode placement; however, the US findings immediately after the ECT have not been previously described. In this study, immediate, intermediate and delayed changes in tumors with US were evaluated for assessment and as an indicator of the appropriate coverage of tumors with a sufficiently strong electric field and predictor of the response of tumors . ${ }^{17,18}$

\section{Patients and methods}

\section{Patients}

Patients were treated by ECT for colorectal liver metastases. The study was approved by the Slovenian National Ethics Committee (\#45/09/08), and registered at ClinicalTrials.gov (NCT02352259;
First Posted Date: February 2, 2015) and conducted according to the Helsinki declaration. The patients signed an informed consent form. Two representative patients of the trial who were treated at the Institute of Oncology in Ljubljana were selected from this study for presentation.

Patient \#1 was a 56-year-old female with a 25 $\mathrm{mm}$ recurrent colorectal liver metastasis located in the left liver lobe under the right hemidiaphragm which was diagnosed in early 2016 . The patient had previously undergone a right hepatectomy and metastasectomy from Sg. II and IV, which was performed in 2009, and sigmoid colon resection in 2006 (T4N1 sigmoid colon adenocarcinoma). Before the first liver operation in 2006, the patient was treated with chemotherapy, which included oxaliplatin, capecitabine and cetuximab.

Patient \#2 was a 59-year-old male diagnosed in 2016 with synchronous, locally advanced rectal cancer and solitary $16 \mathrm{~mm}$ liver metastasis in Sg. VII that was adjacent to the right hepatic vein. He received neoadjuvant treatment, which included chemotherapy (FOLFOX, panitumumab) and a short course of radiation ( 5 x 5 Gy). Previously, he was treated for laryngeal and lung cancer.

\section{Imaging}

Standard pretreatment evaluation of patients with colorectal liver metastases included a liver MRI with a hepatospecific contrast agent and a CT of the thorax and abdomen, including the pelvis at least 1 month before ECT. MRI was performed using a 1.5 T GE Medical Systems Optima MR450w (GE, Chicago, IL) and Siemens Magnetom Avanto syngo MR B17 (Siemens, Erlangen, Germany). CT imaging was performed using contrast-enhanced CT (CECT) on a Siemens SOMATOM Definition AS 64 (Siemens, Erlangen, Germany). The patients were reviewed at a multidisciplinary team. The follow-up assessment included a liver MRI with hepatospecific contrast agent CECT within at least 1-2 months after treatment, after 6 months, and until progression of the disease. In the case of Patient $\# 1$ also follow up using the US was possible, due to the specific location of the lesion. Evaluation of the target lesion and tumor response were measured according to modified RECIST criteria. ${ }^{2}$

ECT was performed under real-time US guidance using a 7.5-MHz linear-array transducer probe on a portable GE ultrasound machine. The follow-up US was performed using an abdominal probe on a Toshiba Aplio 300 ultrasound apparatus (Toshiba, Otawara, Japan). 


\section{Treatment planning}

Treatment planning was performed based on pre-operative images (patient 1: CECT; patient 2: contrast-enhanced T1-weighted MRI). The liver and liver vessels were segmented using semi-automated segmentation algorithms that were developed previously and integrated into the web-based tool, Visifield (www.visifield.com, University of Ljubljana). ${ }^{19,20}$ Electrodes were modelled as conductive cylinders based on commercially available individual needle electrodes (IGEA, Carpi, Italy). ${ }^{9}$ The direction of electrode access was determined by the performing surgeon.

Given the direction of electrodes, a treatment plan was prepared for both patients by optimizing the voltages between four electrodes positioned in a rectangular pattern in the healthy liver parenchyma. The optimization was performed by solving the Laplace equation for the electric potential in tissue using the finite element method. ${ }^{21}$ Since the pulses are delivered to the electrodes in pairs, the computation was performed for each pair of electrodes separately (in total, 6 pairs for 4 electrodes), and the maximum electric field from each pair combined was used to determine the cumulative coverage of a tumor. By sequentially activating the electrodes in this way, a larger tumor volume can be covered with sufficiently strong electric fields. ${ }^{7}$ The goal of the optimization was to ensure a 100\% coverage of the clinical target volume with electric field above $400 \mathrm{~V} / \mathrm{cm}$ and to limit the maximum current delivered to the tissue to be below 50 A (hardware limit of the IGEA Cliniporator Vitae pulse generator). ${ }^{22}$

\section{Electrochemotherapy}

ECT was performed using the same treatment protocol as defined by the SOP for ECT of cutaneous tumors regarding the drug dosage and electrical parameters (i.e. pulse duration and number of pulses) of electroporation..$^{23}$ The procedure was adapted for the treatment of liver tumors during open surgery as previously described. . $^{2,24,25}$

For patient \#1, a transthoracic approach through the diaphragm was chosen due to expected adhesions from previous liver resection and because of the subdiaphragmatic location of the metastasis. Initially, we planned to use long individual needle electrodes and a variable geometry approach (Supplementary data: Table S1, Figure S1); however, during the operation, the plan was changed due to limited space in the thorax. Therefore, a hex- agonal electrode array with 3-cm long needles with fixed geometry was used..$^{9}$ The electrodes were inserted 8 times to cover the entire tumor.

Patient \#2 was operated after neoadjuvant treatment (see above). We planned ECT for him because his liver metastasis was located on the right hepatic vein, which would have required a right hepatectomy simultaneously with a low anterior rectal resection. This was considered to be too extensive of a procedure; therefore, ECT and simultaneous low anterior rectal resection were performed.

The treatment plan was prepared based on the pre-operative MRI or CECT images (Supplementary data: Table S1, Figure S1). The calculation of the optimal electrode placement for specific access was performed. The treatment plan included placing four electrodes located in the safety margin around the tumor and one electrode located centrally in the tumor. The electric pulse amplitudes, which would result in a 100\% coverage of a tumor with electric fields above $400 \mathrm{~V} / \mathrm{cm}$, were calculated (Supplementary data: Table S1).

\section{Reconstruction of the imaging plane}

To attempt to correlate the results of the numerical modelling with observed changes in ultrasound images, the US imaging plane was reconstructed by synthetically generating a 2-D image from the segmented volumetric pre-treatment images with the same dimensions as the US images. The spatial origin and angles of the pseudo-US image were manually adjusted to register the intraoperative US with the anatomical landmarks (tumor location, liver shape and major blood vessels position). ${ }^{26,27}$ To verify this reconstruction, the segmented US images were compared with the reconstructed imaging plane using the Dice-Sørensen coefficient. ${ }^{28}$ The mean value of this coefficient was 0.66 , while the standard deviation was 0.10 .

\section{Results}

ECT was used for the treatment of the liver tumors. US imaging was used for the identification of the electrode placement according to the treatment plan (Supplementary data: Table 1, Figure S1). Two representative cases are presented, and US specific changes are described for identification of adequate tumor coverage with sufficiently strong electric field to ensure electroporation and thus effective ECT. 


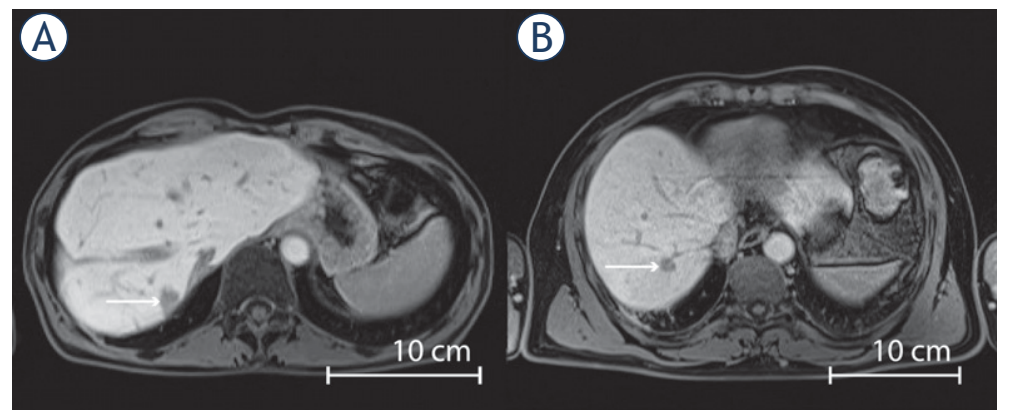

FIGURE 1. Colorectal liver metastases in difficult-to-treat locations. Locations of metastases to be treated with electrochemotherapy are indicated by white arrows (A) subdiafragmally in the liver remnant after right hepatectomy; (B) segment VII near the right hepatic vein.
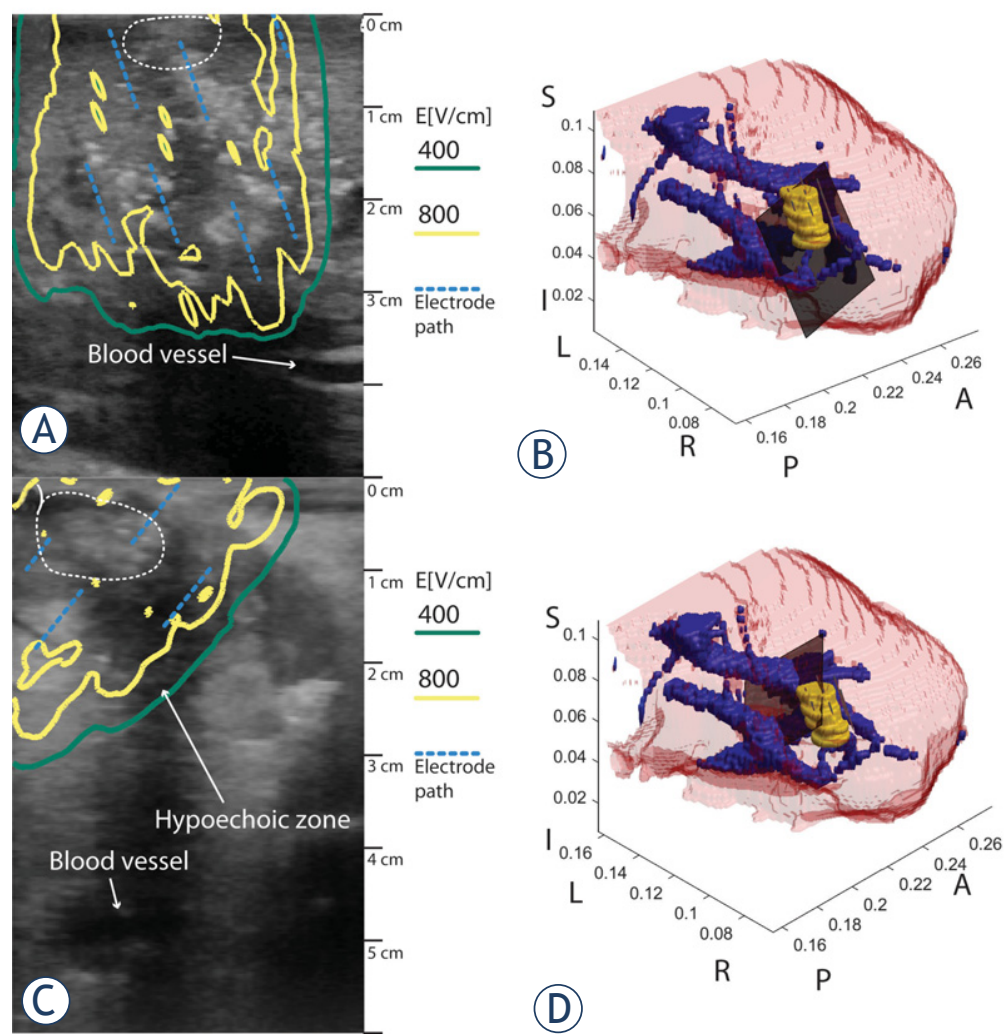

(D)

FIGURE 2. Effects of electrochemotherapy as is visible in the ultrasound imaging. (A) Immediate effect of microbubbles formation in the electrode track (5 min). Location of the metastasis is indicated by the dashed white curve. Overlaid is a contour plot showing iso-contours of the electric field of 400 and $800 \mathrm{~V} / \mathrm{cm}$, yellow and green solid curves, respectively. Electrode locations for one array location are indicated by blue dashed lines (B) llustration of the ultrasound imaging plane in the reconstructed 3-D geometry of the liver. The tumor is shown in yellow, blood vessels are shown in blue, and the liver surface is shown in transparent red. The imaging plane is shown in black. One location of the electrode array is also shown. (C) Microbubbles are resorbed, the hypoechoic area represents the electroporated area, and the hyperechoic tumor is well demarcated within the ablation zone (5-15 min). Location of the metastasis is indicated by dashed white curve. Overlaid are the electric field iso-contours after delivery of all pulses as in (A). (D) llustration of the imaging plane and location. Colors as in (C). Anatomic directions in $(C)$ and $(F)$ are indicated as follows: A - anterior, P - posterior, L - left, R - right, S - superior, I - inferior.

\section{Tumour identification and treatment}

The first case (patient \#1) had liver metastasis of a colorectal tumor that was $20 \mathrm{~mm}$ in diameter, located subdiafragmally in the liver remnant after a right hepatectomy (Figure $1 \mathrm{~A}$ ). Due to its location, we decided to perform ECT with trans-thoracic access using hexagonal electrodes. Therefore, the treatment plan was not executed as originally foreseen.

The second case (patient \#2) had liver metastasis of a colorectal tumor in segment VII that was near the right hepatic vein and $19 \mathrm{~mm}$ in diameter (Figure $1 \mathrm{~B}$ ). Due to its location, we decided to perform ECT using long needle electrodes. The placement of the electrodes was US-guided and confirmed to be close to the treatment plan.

\section{Ultrasonographic findings following ECT}

The ablation zone was monitored by the US immediately after delivery of electric pulses during the ECT session. In patient \#1, it was also possible to monitor the outcome using US few days and also several months after ECT and compare it to MRI due to the tumor's location and trans-thoracic approach. Based on US observations of immediate effects, the tumor coverage with a sufficiently high electric field can be predicted, whereas the intermediate effects can confirm adequate coverage of the tumor with an effective electric field. The late effects indicated the progressive resorption of the treated zone within a few months after efficient ECT.

\section{Immediate effects}

Immediate effects of ECT were observed after delivery of the first electric pulses and up to 1.5 $\mathrm{h}$ thereafter. The observed US changes were followed in the ablation zone to identify whether they appear in the entire treated tumor for possible indication of effective electroporation of the tumor. The observed changes can be further divided into two phases that were observed in both patient \#1 and patient \#2.

The $1^{\text {st }}$ phase took up to 5 minutes after the delivery of electric pulses. The hyperechoic microbubbles were observed along the electrode tracks and were visible immediately i.e. within a few seconds after the pulses were triggered and later within the entire ablation zone (Figure $2 \mathrm{~A}$ ).

During the $2^{\text {nd }}$ phase, after 5-15 min, microbubbles were distributed throughout the treated tumor, and the tumor became hyperechoic and surrounded 
by a hypoechoic zone. The hypoechoic zone (5-15 $\mathrm{mm}$ wide) represents the electroporated area within the normal liver tissue represents the treatment safety margin. Because the tumor within this hypoechoic area is differentiated as hyperechoic, this approach can predict adequate coverage of the treated tumor with an adequate electric field, when the hyperechogenicity is evenly distributed throughout an ECT-treated tumor (Figure 2 C). To confirm this finding, the ablation zone was correlated with the placement of the electrodes. The hypoechoic area coincided with the predicted area that needed to be treated for complete coverage of a tumor for its effective ablation (Figure $2 \mathrm{~A}, \mathrm{C}$ ).

Similar findings were also observed in the second case (patient \#2), where long individual needle electrodes were used for the variable electrode configuration. The electrode track was already observed few seconds after electroporation (Figure 3 A) followed by hypoechoic changes and microbubble formation in the $2^{\text {nd }}$ phase after 5-15 minutes (Figure $3 \mathrm{C}$ ). This patient underwent another surgical procedure within the same operation after ECT. This enabled the investigation of the ablation zone $1.5 \mathrm{~h}$ after ECT (Figure $3 \mathrm{E}$ ). The hypoechoic ablation zone was visible with a clearly demarcated and evenly hyperechoic tumor. Based on the observation in patient $\# 1$, we assumed that the tumor was adequately electroporated with a sufficient safety margin. Again, the hypoechoic area coincided with the predicted area that needed to be treated for complete coverage of the tumor for its effective eradication (Figure $3 \mathrm{~A}, \mathrm{C}, \mathrm{E}$ ).

\section{Intermediate effects as observed by US after a few days}

Four days after ECT US of the metastasis in patient \#1 she presented with an 18-mm hyperechoic formation surrounded by a 5-mm hypoechoic area, which is most likely the oedematous area of the liver parenchyma, or the safety margin (Figure 4). The metastasis had a similar appearance as immediately after the ECT. In this case, transabdominal US imaging of the ECT-treated metastasis was possible due to the specific location and the transthoracic approach, which did not result in the air in the abdominal cavity.

\section{Late effects}

The response to the treatment was evaluated 5 months after the treatment by US and compared to the MRI in patient \#1. This evaluation was possible due to the specific location of the metastasis, which also enabled the verification of its appearance by
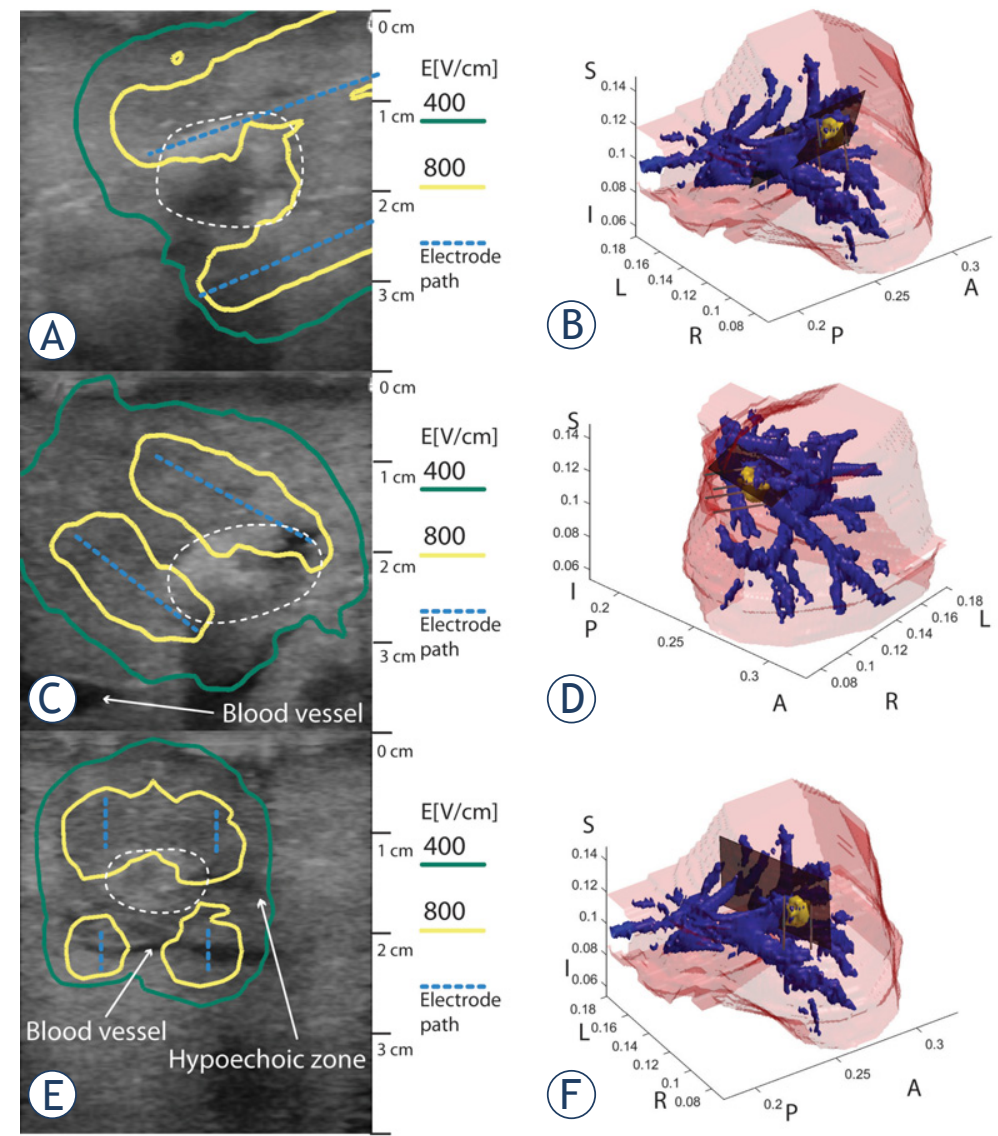

FIGURE 3. Effects of electrochemotherapy (ECT) as visible in the US imaging. (A) Immediate effect of microbubble formation in the electrode track immediately after electroporation ( $5 \mathrm{~min}$ ). Location of the metastasis is indicated by the dashed white curve. Overlaid is a contour plot showing iso-contours of the electric field of 400 and $800 \mathrm{~V} / \mathrm{cm}$, yellow and green solid curves, respectively. Electrode locations are indicated by dashed blue lines (B) Illustration of the US imaging plane in the reconstructed 3-D geometry of the liver. The tumor is shown in yellow, blood vessels in blue, and the liver surface in transparent red. The imaging plane is shown in black. (C) First hyperechoic changes and microbubbles after treatment (5-15 minutes). Location of the metastasis is indicated by dashed curve. Overlaid are the electric field iso-contours. Colors as in (A). (D) Illustration of the imaging plane and location. Colors as in (B). (E) Hyperechoic tumor in the hypoechoic ablation zone $1.5 \mathrm{~h}$ after ECT. Location of the metastasis is indicated by dashed white curve. Electric field and electrodes are shown as above. (F) Illustration of the imaging plane and location. Colors as in (B). Anatomic directions in (B), (D), and (F) are indicated as follows: A anterior, $\mathrm{P}$ - posterior, $\mathrm{L}$ - left, $\mathrm{R}$ - right, $\mathrm{S}$-superior, I - inferior.

US. Five months after ECT, US and MRI showed the metastasis in patient $\# 1$ as a fibrotic residuum without the hypoechoic rim. The size of the metastasis was not significantly reduced (Figure 5 A, B). The appearance was as a complete response, which was also present 7 months after ECT; however, at that time, the size was significantly reduced to 11 $\mathrm{mm}$ from the original $20 \mathrm{~mm}$ in the greatest diameter, indicating the slow resorption of the treated metastasis. Similar MRI findings were shown in patient \#2, 3 months after ECT in which the treated 


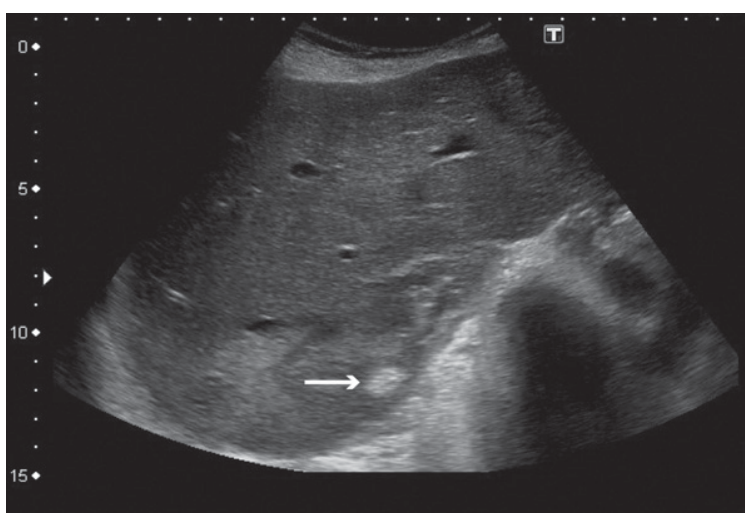

FIGURE 4. Transabdominal US of liver metastasis 4 days after electrochemotherapy. The metastasis was well demarcated and hyperechoic (indicated by the white arrow) in the hypoechoic ablation zone.

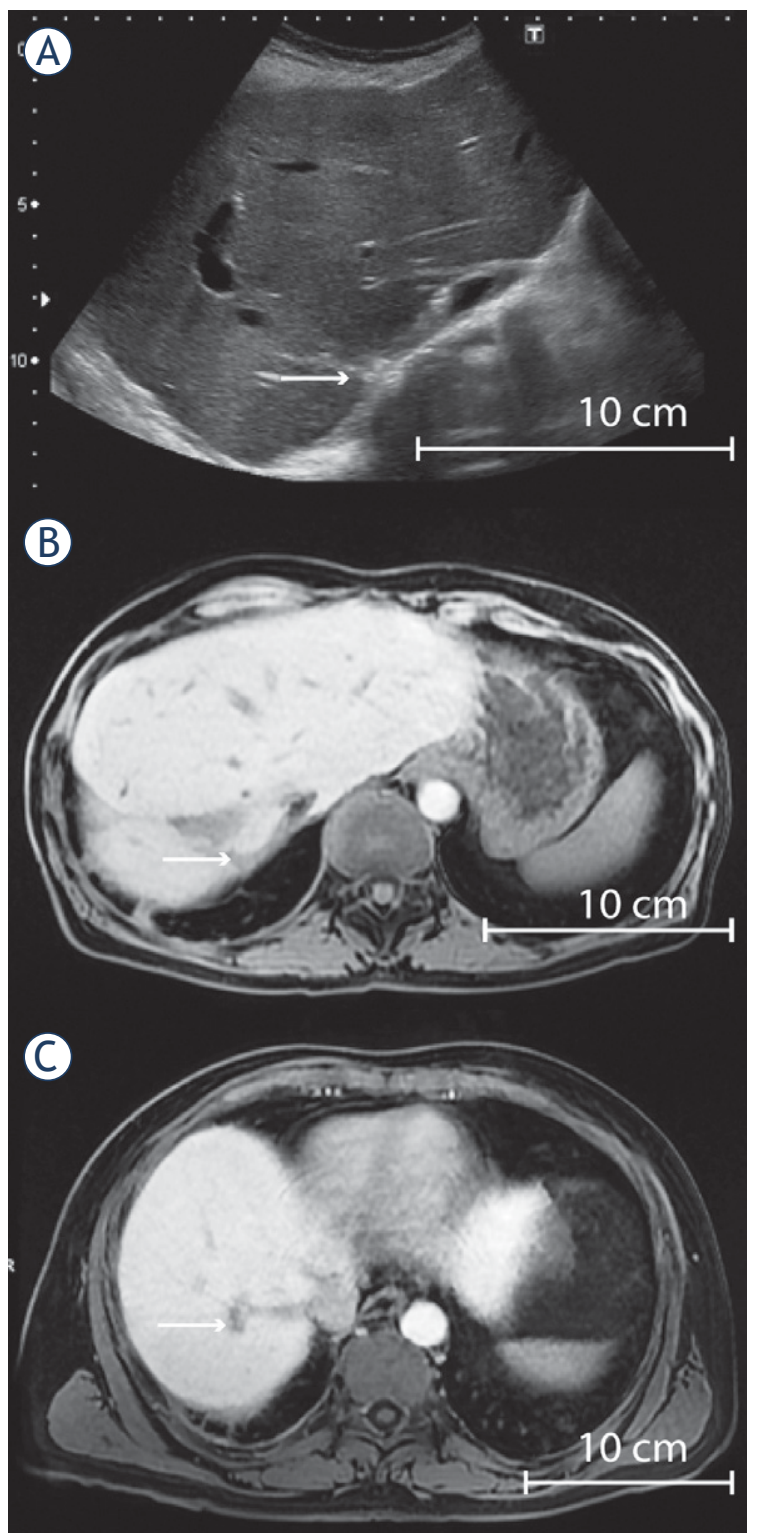

metastasis was reduced from 19 to $13 \mathrm{~mm}$ in diameter (Figure $5 \mathrm{C}$ ).

\section{Discussion}

Monitoring changes in the tissue during and after ablative techniques, including ECT, is important to evaluate the progress of the procedure and its result. It is important not to overestimate or underestimate the ablative zone because both have their drawbacks. Therefore, treatment monitoring and understanding the imaging findings to predict the tumor response to ECT are important.

We observed that hyperechoic foci are indicators of the electroporated tissue (tumor), and when the area coincides with the whole tumor mass, it indicates adequate tumor coverage with sufficiently strong electric field. The hyperechogenic microbubbles that form around the electrodes are a consequence of electrochemical reactions on the electrodes. ${ }^{29-32}$ These are gaseous bubbles that then disperse throughout the tumors. Monitoring the hyperechogenicity of the tumors is thus important for monitoring the treatment of liver tumors during intraoperative ECT, where US helps not only with proper electrode placement but also has an important role in assessment and as an indicator of the tumor coverage with a sufficiently strong electric field. This is important for the electroporation of the whole tumor to facilitate bleomycin entry into the tumor cells, which can then exert its potent cytotoxic effect on cells. ${ }^{33}$ Around the tumour and in the normal tissue, a hypoechogenic rim is formed, indicating tissue edema. This observation is due to the blockade of the tumor perfusion due to the electric pulse application and water leakage out of the tissue. ${ }^{34}$ The formation of the safety halo around a tumour further supports the presumption that a tumour was adequately covered by the effective electric field during the ECT (Figure 6).

Treatment planning for ECT and IRE is gaining in its importance ${ }^{1}$, specifically with a new webbased tool that will enable the spread of this approach. ${ }^{20}$ Treatment planning based on numerical methods for computing the electric field is the best

FIGURE 5. Late effects of electrochemotherapy (ECT). (A) US image 5 months after ECT in patient \#1. (B) MRI 5 months after ECT in patient \# 1. Metastasis of reduced size presented as a fibrotic residuum without the edematous rim. (C) MRI 3 months after ECT in patient \#2. The metastasis of reduced size presented as a fibrotic residuum without the edematous rim. All lesions are indicated by white arrows. 
tool for verifying the feasibility of electroporationbased treatments and determining the optimal electrode placement for successful coverage of the entire tumor during treatment in advance of the treatment. The placement of the electrodes is critical, since placement errors can lead to insufficient coverage of the target tissue. ${ }^{18}$ Coupling with stereotactic navigation or optical navigation has been shown to be feasible, and CT guidance is often used for percutaneous IRE treatments. ${ }^{35-37}$ However, in situations when the placing of the electrodes cannot follow the treatment plan, e.g., due to tumour growth between imaging and the time of the treatment, poor contrast of the tumour in pre-treatment imaging or unforeseen difficulties in placing the electrodes according to the treatment plan, a tool to verify the coverage of the tumour with sufficient electric fields is important. MRI imaging has been proposed for monitoring the electric field during pulse delivery; however, the clinical application still requires further developments, such as MRIcompatible pulse generators and electrodes. ${ }^{15,16}$ US thus lends itself as a tool for monitoring the immediate tissue response: the appearance of hyperechoic and hypoechoic changes are possibly indicative of tissue electroporation, and when the area coincides with the treated tumor and the treatment plan, as shown in this study, then the complete response of the treated tumor can be anticipated.

Interestingly, the US findings are comparable with the MRI findings - a hyperechoic tumor with a hypoechoic rim, which was shown to be the safety margin. Most likely, the hypoechoic rim represents the edematous normal tissue, which gradually resorbs after electroporation/pulse delivery, as shown also in this study. This could also be related to the differential effect of ECT on the normal versus tumor tissue vasculature, which has been demonstrated in a mouse model using window chamber microscopy. ${ }^{38,39}$

US has already been used for the observation of tissue changes after IRE in the study on pigs. In the acute period, a hypoechoic area with well-demarcated margins appeared. Therefore, the ablation zone can be predicted by measuring the external hyperechoic rim that forms 90-120 minutes after IRE. The rim is possibly attributable to evolving hemorrhagic infiltration via widened sinusoids. ${ }^{40}$ Our study demonstrates similar findings after reversible electroporation, though in the ECT treated cancer patients.

The response of a tumour after ECT is slow due to its mode of action, i.e., due to slow killing of the dividing cells that occurs due to the internalization

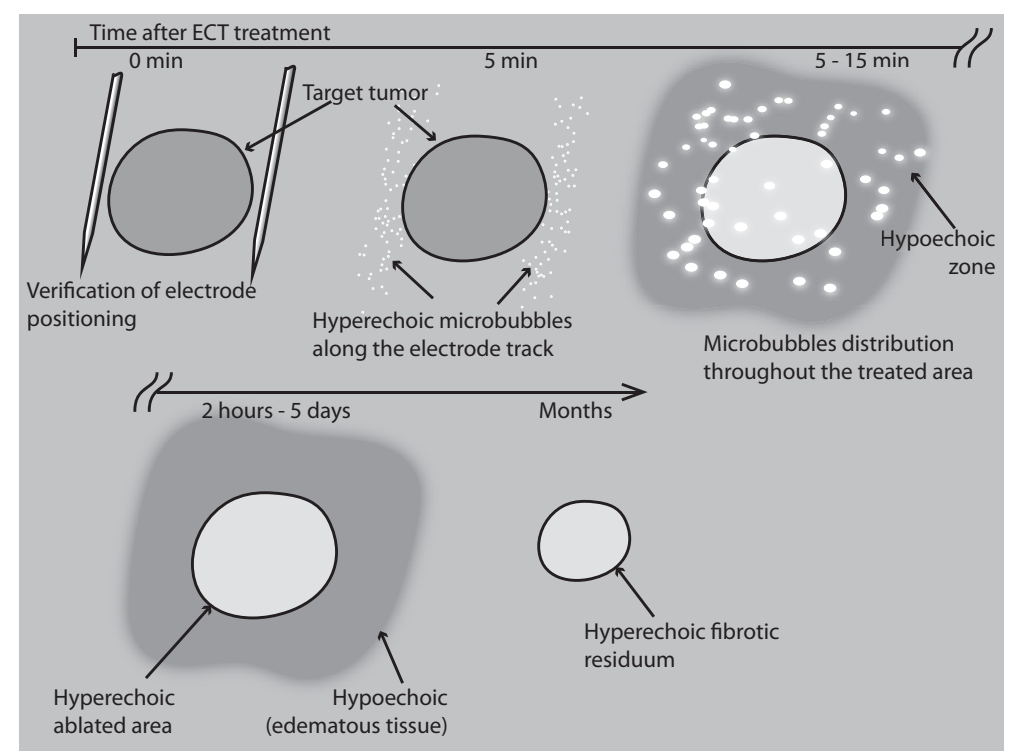

FIGURE 6. Schematic representation of the timeline of ultrasonographic changes after the delivery of electrochemotherapy treatment.

of the bleomycin by electroporation. ${ }^{41,42}$ The treated tumor gradually changes into fibrotic tissue during a period of 4 months, as shown in the MRI images. Later, the tumor progressively shrinks with slow resorption of the necrotic tumor tissue and fibrosis. ${ }^{42}$

In conclusion, we have described US post-ECT changes that can serve as an indicator of tumor coverage with a sufficiently strong electric field and a predictor of the response of the tumors. The US findings during the treatment are comparable with the MRI findings during the follow-up period.

\section{Acknowledgments}

The authors would like to thank Tjaša Maslo, Andrej Vogrin, and Marija Brelih for assisting with segmentation of the ultrasound images. The authors acknowledge the financial support from the state budget for the Slovenian Research Agency (ARRS) under various grants (P3-0003, P2-0249, and Z3-7126). The research was conducted in the scope of LEA EBAM (French-Slovenian European Associated Laboratory: Pulsed Electric Fields Applications in Biology and Medicine).

\section{Data availability}

The data pertaining to this manuscript are available at the figshare repository under DOI: 10.6084/ m9.figshare.5422858. 


\section{Author contribution statement}

$\mathrm{NB}, \mathrm{IE}, \mathrm{MM}, \mathrm{BT}$ and GS conceived and designed the experiments, $\mathrm{BK}, \mathrm{NB}, \mathrm{IE}, \mathrm{EB}, \mathrm{BT}, \mathrm{MB}$ and $\mathrm{MD}$ performed the experiments; $\mathrm{BK}, \mathrm{NB}, \mathrm{MM}$ analyzed the data; GS, BK, NB, MC and DM wrote the manuscript; BK prepared the figures. All authors read and approved the final version of the manuscript.

\section{References}

1. Miklavcic D, Davalos RV. Electrochemotherapy (ECT) and irreversible electroporation (IRE) -advanced techniques for treating deep-seated tumors based on electroporation. Biomed Eng Online 2015; 14: 11. doi: 10.1186/1475-925X-14-S3-I1

2. Edhemovic I, Brecelj E, Gasljevic G, Marolt Music M, Gorjup V, Mali B, et al. Intraoperative electrochemotherapy of colorectal liver metastases. J Surg Oncol 2014; 110: 320-7. doi: 10.1002/jso.23625

3. Rubinsky B. Irreversible electroporation in medicine. Technol Cancer Res Treat 2007; 6: 255-60. doi: 10.1007/978-3-642-05420-4

4. Chapiro J, Geschwind J-F. Science to practice: systemic implications of ablative tumor therapies - reality uncovered and myths exposed? Radiology 2016; 280: 329-31. doi: 10.1148/radiol.2016160505

5. Coletti L, Battaglia V, De Simone P, Turturici L, Bartolozzi C, Filipponi F. Safety and feasibility of electrochemotherapy in patients with unresectable colorectal liver metastases: a pilot study. Int J Surg 2017; 44: 26-32. doi: 10.1016/j.ijsu.2017.06.033

6. Campana LG, Clover AJP, Valpione S, Quaglino P, Gehl J, Kunte C, et al Recommendations for improving the quality of reporting clinical electrochemotherapy studies based on qualitative systematic review. Radiol Oncol 2016: 50: 1-13. doi: 10.1515/raon-2016-0006

7. Yarmush ML, Golberg A, Serša G, Kotnik T, Miklavčič D. Electroporationbased technologies for medicine: principles, applications, and challenges. Annu Rev Biomed Eng 2014; 16: 295-320. doi: 10.1146/annurev-bioeng-071813-104622

8. Chunlan J, Davalos RV, Bischof JC. A review of basic to clinical studies of irreversible electroporation therapy. Biomed Eng IEEE Trans 2015; 62: 4-20. doi: 10.1109/TBME.2014.2367543

9. Miklavčič D, Mali B, Kos B, Heller R, Serša G. Electrochemotherapy: from the drawing board into medical practice. Biomed Eng Online 2014; 13: 29. doi: $10.1186 / 1475-925 X-13-29$

10. Edhemovic I. Electrochemotherapy: a new technological approach in treatment of metastases in the liver. Technol Cancer Res Treat 2011; 10: 475-85. doi: 10.7785/tcrt.2012.500224

11. Edhemovic I, Brecelj E. Electrochemotherapy of liver tumors: colorecta liver metastasis. In: Vernier PT, Rubinsky B, Juergen F. Miklavcic D, Rols MP, Raso J, et al, editors. Handbook of electroporation. Springer International Publishing; 2016. p. 1-12. doi: 10.1007/978-3-319-26779-1_98-1

12. Djokic M, Cemazar M, Popovic P, Kos B, Dezman R, Bosnjak M, et al. Electrochemotherapy as treatment option for hepatocellular carcinoma, a prospective pilot study. EJSO 2018; 44: 651-7. doi: 10.1016/j. ejso.2018.01.090

13. Neal II RE, Cheung W, Kavnoudias $\mathrm{H}$, Thomson KR. Spectrum of imaging and characteristics for liver tumors treated with irreversible electroporation. $J$ Biomed Sci Eng 2012; 05: 813-8. doi: 10.4236/jbise.2012.512A102

14. Zhang Y, Guo Y, Ragin AB, Lewandowski RJ, Yang G-Y, Nijm GM, et al. MR Imaging to assess immediate response to irreversible electroporation for targeted ablation of liver tissues: Preclinical feasibility studies in a rodent model. Radiology 2010; 256: 424-32. doi: 10.1148/radiol.10091955

15. Kranjc M, Kranjc S, Bajd F, Serša G, Serša I, Miklavčič D. Predicting irreversible electroporation-induced tissue damage by means of magnetic resonance electrical impedance tomography. Sci Rep 2017; 7: 10323. doi: 10.1038/ s41598-017-10846-5
16. Kranjc M, Markelc B, Bajd F, Čemažar M, Serša I, Blagus T, et al. In situ monitoring of electric field distribution in mouse tumor during electroporation. Radiology 2015; 274: 115-23. doi: 10.1148/radiol.14140311

17. Miklavcic D, Corovic S, Pucihar G, Pavselj N. Importance of tumour coverage by sufficiently high local electric field for effective electrochemotherapy. EJC Supplements 2006; 4: 45-51. doi: 10.1016/j.ejcsup.2006.08.006

18. Miklavcic D, Snoj M, Zupanic A, Kos B, Cemazar M, Kropivnik M, et al. Towards treatment planning and treatment of deep-seated solid tumors by electrochemotherapy. Biomed Eng Online 2010; 9: 10. doi: 10.1186/1475925X-9-10

19. Marčan M, Kos B , Miklavčič D. Effect of blood vessel segmentation on the outcome of electroporation-based treatments of liver tumors. PLoS One 2015; 10: e0125591. doi: 10.1371/journal.pone.0125591

20. Marčan M, Pavliha D, Kos B, Forjanič T, Miklavčič D. Web-based tool for visualization of electric field distribution in deep-seated body structures and planning of electroporation-based treatments. Biomed Eng Online 2015; 14 Suppl 3: S4. doi: 10.1186/1475-925X-14-S3-S4

21. Zupanic A, Kos B, Miklavcic D. Treatment planning of electroporationbased medical interventions: electrochemotherapy, gene electrotransfer and irreversible electroporation. Phys Med Biol 2012; 57: 5425-40. doi: 10.1088/0031-9155/57/17/5425

22. Cliniporator BC. Medical electroporation of tumors. In: Vernier PT, Rubinsky B, Juergen F. Miklavcic D, Rols MP, Raso J, et al, editors. Handbook of electroporation. Springer International Publishing; 2017. p. 1-36. doi: 10.1007/978-3-319-26779-1 214-1

23. Gehl J, Sersa G, Matthiessen LW, Muir T, Soden D, Occhini A, et al. Updated standard operating procedures for electrochemotherapy of cutaneous tumours and skin metastases. Acta Oncol 2018; 57: 874-82. doi: $10.1080 / 0284186 \times .2018 .1454602$

24. Edhemovic I, Gadzijev EM, Brecelj E, Miklavcic D, Kos B, Zupanic A, et al. Electrochemotherapy: a new technological approach in treatment of metastases in the liver. Technol Cancer Res Treat 2011; 10: 475-85. doi: 10.1109/ TNB.2011.2128340

25. Mali B, Gorjup V, Edhemovic I, Brecelj E, Cemazar M, Sersa G, et al. Electrochemotherapy of colorectal liver metastases - an observational study of its effects on the electrocardiogram. Biomed Eng Online 2015; 14 Suppl 3: S5. doi: 10.1186/1475-925X-14-S3-S5

26. De Buck S, Maes F, Ector J, Bogaert J, Dymarkowski S, Heidbüchel H, et al. An augmented reality system for patient-specific guidance of cardiac catheter ablation procedures. IEEE Trans Med Imaging 2005; 24: 1512-4. doi: 10.1109/TMI.2005.857661

27. King AP, Rhode KS, Ma Y, Yao C, Jansen C, Razavi R, et al. Registering preprocedure volumetric images with intraprocedure 3-D ultrasound using an ultrasound imaging model. IEEE Trans Med Imaging 2010; 29: 924-37. doi: 10.1109/TMI.2010.2040189

28. Zijdenbos AP, Dawant BM, Margolin RA, Palmer AC. Morphometric analysis of white matter lesions in MR images: method and validation. IEEE Trans Med Imaging 1994; 13: 716-24. doi: 10.1109/42.363096

29. Saulis G, Lapè R, Pranevičiūtè R, Mickevičius D. Changes of the solution pH due to exposure by high-voltage electric pulses. Bioelectrochemistry 2005 67: 101-8. doi: 10.1016/j.bioelechem.2005.03.001

30. Maglietti F, Michinski S, Olaiz N, Castro M, Suárez C, Marshall G. The role of Ph fronts in tissue electroporation based treatments. PLoS One 2013; 8: e80167. doi: 10.1371/journal.pone.0080167

31. Stehling MK, Guenther E, Mikus P, Klein N, Rubinsky L, Rubinsky B. Synergistic combination of electrolysis and electroporation for tissue ablation. PLoS One 2016; 11: e0148317. doi: 10.1371/journal.pone.0148317

32. Rubinsky L, Guenther E, Mikus P, Stehling M, Rubinsky B. Electrolytic effects during tissue ablation by electroporation. Technol Cancer Res Treat 2016; 15: NP95-NP103. doi: 10.1177/1533034615601549

33. Orlowski S, Mir LM. Cell electropermeabilization: a new tool for biochemical and pharmacological studies. Biochim Biophys Acta 1993; 1154: 51-63.

34. Jarm T, Cemazar M, Miklavcic D, Sersa G. Antivascular effects of electrochemotherapy: implications in treatment of bleeding metastases. Expert Rev Anticancer Ther 2010; 10: 729-46. doi: 10.1586/era.10.43 
35. Groselj A, Kos B, Cemazar M, Urbancic J, Kragelj G, Bosnjak M, et al. Coupling treatment planning with navigation system: a new technological approach in treatment of head and neck tumors by electrochemotherapy. Biomed Eng Online 2015; 14 Suppl 3: S2. doi: 10.1186/1475-925X-14-S3-S2

36. Narayanan G, Hosein PJ, Beulaygue IC, Froud T, Scheffer HJ, Venkat SR, et al. Percutaneous image-guided irreversible electroporation for the treatment of unresectable, locally advanced pancreatic adenocarcinoma. J Vasc Interv Radiol 2017; 28: 342-48. doi: 10.1016/j.jvir.2016.10.023

37. Beyer LP, Pregler B, Michalik K, Niessen C, Dollinger M, Müller M, et al. Evaluation of a robotic system for irreversible electroporation (IRE) of malignant liver tumors: initial results. Int J Comput Assist Radiol Surg 2017; 12: 803-9. doi: 10.1007/s11548-016-1485-1

38. Markelc B, Sersa G, Cemazar M. Differential mechanisms associated with vascular disrupting action of electrochemotherapy: Intravital microscopy on the level of single normal and tumor blood vessels. PLoS One 2013; 8: e59557. doi: 10.1371/journal.pone.0059557

39. Granata V, Fusco R, Setola SV, Piccirillo M, Leongito M, Palaia R, et al. Early radiological assessment of locally advanced pancreatic cancer treated with electrochemotherapy. World J Gastroenterol 2017; 23: 4767-78 doi: 10.3748/wjg.v23.i26.4767

40. Appelbaum L, Ben-David E, Sosna J, Nissenbaum Y, Goldberg SN. US Findings after irreversible electroporation ablation: Radiologic-pathologic correlation. Radiology 2012; 262: 117-25. doi: 10.1148/radiol.11110475

41. Orlowski S, Belehradek J, Paoletti C, Mir LM. Transient electropermeabilization of cells in culture. Increase of the cytotoxicity of anticancer drugs. Biochem Pharmacol 1988; 37: 4727-33. doi: 10.1016/0006-2952(88)903449

42. Gasljevic G, Edhemovic I, Cemazar M, Brecelj E, Gadzijev EM, Music MM, et al. Histopathological findings in colorectal liver metastases after electrochemotherapy. PLoS One 2017; 12: e0180709. doi: 10.1371/journal. pone.0180709 